\title{
Fratura proximal de tíbia após cirurgia extracapsular para correção de ruptura de ligamento cruzado cranial em cão
}

\author{
Tibial fracture caused by complications after an extracapsular suture for the repair of cranial cruciate \\ ligament rupture in a dog
}

\author{
Jaqueline França dos Santos ${ }^{\mathrm{I}}$ Cássio Ricardo Auada Ferrigno ${ }^{\mathrm{II}}$ Márcio Poletto Ferreira ${ }^{\mathrm{I}}$ \\ Olicies da Cunha ${ }^{\mathrm{I}}$ Kelly Cristiane Ito ${ }^{\mathrm{I}}$ Vanessa Couto de Magalhães Ferraz ${ }^{\mathrm{I}}$ \\ Daniela Fabiana Izquierdo Caquias ${ }^{\mathrm{I}}$ Marcos Ishimoto Della Nina ${ }^{\mathrm{I}}$ Adriana Valente Figueiredo \\ Alexandre Navarro Alves de Souza
}

\section{RESUMO}

Ruptura de ligamento cruzado cranial (RLCCr) é a afecção ortopédica mais frequente no joelho de cães e a sutura fabelo-tibial é frequentemente utilizada na sua correção. Essa técnica pode estar associada com complicações incisionais, lesão tardia de menisco, edema associado ao material de sutura $e$ infecção. $O$ objetivo desta nota foi relatar a ocorrência de fratura proximal de tíbia em um cão, causada por complicação em sutura fabelo-tibial para correção de RLCCr. Um canino atendido em outro serviço foi submetido à osteossíntese com placa 2,0mm para correção de complicação em técnica extracapsular, na qual houve fratura da tíbia na região do orifício criado para confecção da sutura fabelo-tibial. Houve falha dos implantes e a placa foi removida. A fratura foi estabilizada com fixador esquelético externo circular, sendo constatada, após 120 dias, consolidação.

Palavras-chave: sutura fabelo-tibial, complicação, ortopedia, joelho.
ABSTRACT
Cranial cruciate ligament rupture (CCLR) is one of the most common orthopedic diseases in dogs and extracapsular suture with nylon is often used for its correction. This technique may be associated with incisional complications, post-surgical meniscus injury, swelling associated with suture material and infection. The objective of this study is to report a tibial fracture in a dog, caused by complications after an extracapsular suture for the repair of CCLR. An adult dog, which suffered a tibial fracture after an extracapsular fixation for a CCLR, underwent surgery for the fixation of the fracture

with a $2.0 \mathrm{~mm}$ plate, by a referring veterinarian, but the implant failed. The plate was removed and the fracture was stabilized with a circular fixator. Bone consolidation was observed after 120 days.

Key words: fibular-tibial extracapsular suture, complications, orthopedic, stifle.

A ruptura de ligamento cruzado cranial (RLCCr) é considerada uma das afecções ortopédicas mais frequentes em cães e, segundo JOHNSON et al. (1994), é a principal causa de claudicação com origem no joelho. Pode afetar cães de diferentes idades e tamanhos, porém, é mais comum nas raças de grande porte (CASALE \& MCCARTHY, 2009). O reparo do ligamento cruzado cranial tem como objetivo promover estabilidade na articulação do joelho (BUQUERA et al., 2007). As técnicas cirúrgicas para correção da RLCCr em cães incluem estabilização com técnicas de osteotomia (KIM et al., 2008), procedimentos intraarticulares (WARZEE et al., 2001) e extra-articulares (GAMBARDELLA et al., 1981), porém, ainda não foi estabelecida qual técnica é superior (WARZEE et al., 2001). Os procedimentos extra-capsulares têm como vantagens o menor tempo cirúrgico, mínima invasão articular e melhor resposta clínica e, dentre estas, a

\footnotetext{
'Programa de Pós-graduação em Clínica Cirúrgica Veterinária, Universidade de São Paulo (USP), São Paulo, SP, Brasil.

"Departamento de Cirurgia, Faculdade de Medicina Veterinária e Zootecnia (FMVZ), USP, Rua Orlando de Marques Paiva, 87, 05508-270, Cidade Universitária, São Paulo, SP, Brasil. E-mail: cassioaf@usp.br. Autor para correspondência.
} 
técnica menos exigente é a sutura fabelo-tibial (SFT) (SMITH, 2000). Segundo CASALE \& MCCARTHY (2009), a SFT está associada a menos complicações trans e pós-operatórias, mas pode apresentar desvantagens como compressão excessiva das superfícies articulares e soltura, afrouxamento ou rompimento do fio (SMITH, 2000), sendo possível observar deslocamento distal do sesamoide poplíteo (BUQUERA et al., 2007). Foi relatada como complicação da SFT a reação ao material de sutura em 18 a $21 \%$ entre 66 pacientes avaliados (DULISCH, 1981). Em um estudo que avaliou 363 cirurgias de SFT em 305 cães, foram observadas 65 complicações em 63 procedimentos, dentre elas, avulsão da fabela (1), déficit neurológico (1), complicações incisionais (32), lesão tardia do menisco (7), edema associado ao material de sutura (10) e infecção (14), não sendo observada fratura de tíbia como complicação de SFT (CASALE \& MCCARTHY, 2009).

Em fraturas de tíbia, uma das opções para tratamento é o fixador esquelético externo circular (FEEC) que neutraliza as forças de torção e arqueamento, mas permite micro movimentação axial (LEWIS et al., 1998). Essa técnica tem sido utilizada em Medicina Veterinária há pelo menos 15 anos (HALLING et al., 2004), inclusive no tratamento de não-união de fraturas (OWEN, 2000). Quando possível, deve-se utilizar dois anéis em cada fragmento (BILGILI, 2004), porém, em cães de raças pequenas, pode ser aplicado apenas um anel em cada fragmento da fratura (OLCAY \& BILGILI, 1999). O objetivo deste trabalho é relatar ocorrência de fratura proximal de tíbia após técnica cirúrgica de sutura fabelo- tibial para correção de RLCCr, estabilizada com fixador esquelético externo circular em cão.

Um cão da raça Yorkshire Terrier, macho, com oito anos de idade foi atendido no Laboratório de Ortopedia e Traumatologia Comparada do Hospital Veterinário da Universidade de São Paulo (LOTCHOVET-USP) com histórico de RLCCr havia dois meses, tratada com sutura fabelo tibial por Médico Veterinário externo ao LOTC. Nesse procedimento, foi utilizado fio de náilon monofilamentar, sendo este ancorado na fabela lateral e em dois pontos (orifícios) criados na tíbia, um na porção distal da crista da tíbia e o outro $5 \mathrm{~mm}$ distal a este. Após 45 dias da cirurgia, houve fratura da tíbia na região do orifício (distal) realizado para passagem do fio (Figura 1A). A fratura foi corrigida com placa 2,0mm/5 furos, porém, houve falha do implante / técnica (Figura 1B). No exame clínico, foi verificada impotência funcional do membro pélvico esquerdo, dor, crepitação, e, no exame radiográfico, foi observada falha dos implantes metálicos e deslocamento dos fragmentos da fratura.

O paciente foi operado e a fratura estabilizada com FEEC composto de semi-anel proximal e dois anéis $(50 \mathrm{~mm} \varnothing)$ distais à fratura, com dois pinos olivados $(1,5 \mathrm{~mm} \varnothing)$ por anel, estabilizando a fratura de tíbia (Figura 1C). Quatro dias após a cirurgia, o animal apresentava apoio parcial do membro. Foi realizada compressão da fratura $(1 \mathrm{~mm})$ nos dias quatro e 17 de pós-operatório. Com três meses após a cirurgia, o animal apresentava deambulação normal e, com 120 dias de pós-operatório PO, foi constatada consolidação da fratura e o FEEC foi removido (Figura 1D e E). Foi

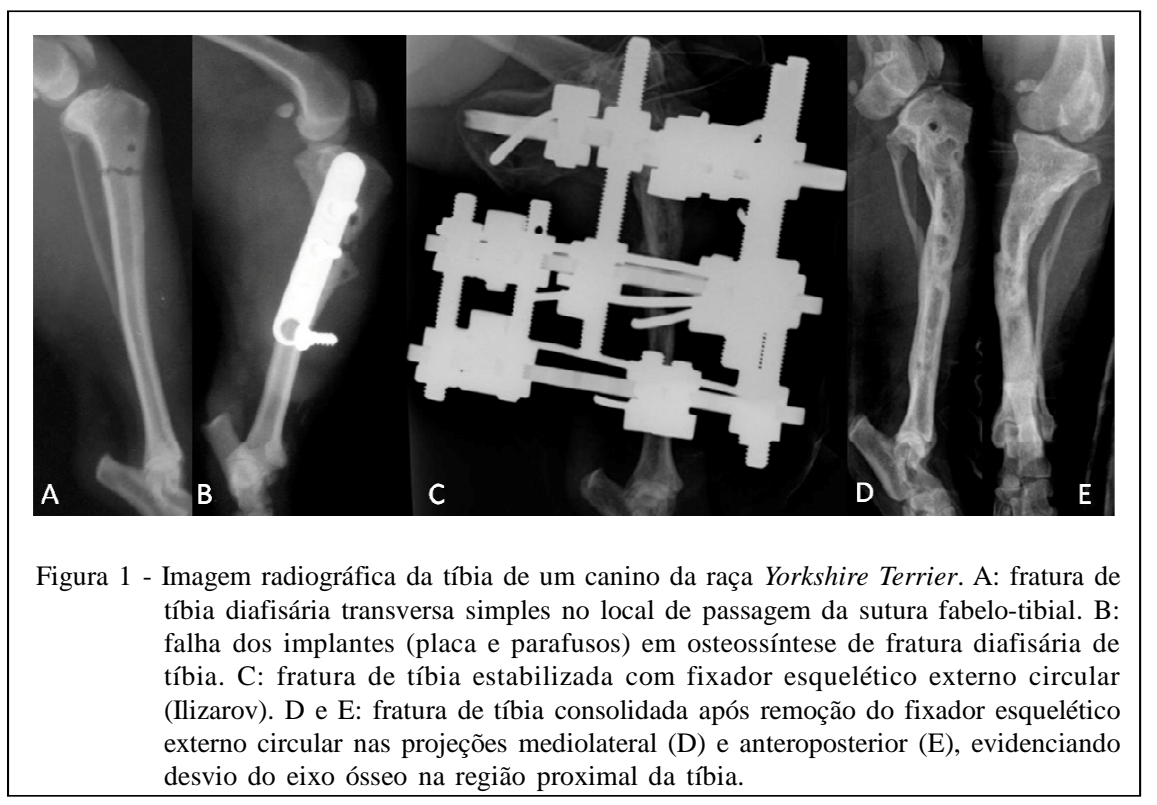

Ciência Rural, v.43, n.3, mar, 2013. 
observado, no exame radiográfico, após a remoção do FEEC, desvio do eixo ósseo em terço proximal de tíbia, porém este não impossibilitava deambulação normal e o membro manteve-se funcional em todas as atividades do animal.

A SFT utilizada na correção da RLCCr no cão deste relato é a técnica extra-articular mais comumente utilizada e tem se mostrado eficiente na estabilização da articulação do joelho, principalmente em cães de pequeno porte (KORVICK et al., 1994) com resultado bom ou excelente em 77 a $82 \%$ dos casos (CHAUVET et al., 1996). Existem poucos relatos de complicações relacionadas à SFT (CASALE \& MCCARTHY, 2009), entre elas estão reação ao material de sutura (DULISCH, 1981), avulsão da fabela, déficit neurológico, complicações incisionais, lesão tardia do menisco, edema associado ao material de sutura e infecção (CASALE \& MCCARTHY, 2009), não tendo sido relatada fratura de tíbia no local de passagem do fio da SFT como complicação da técnica.

Estas complicações tendem a aumentar em cães de maior peso e atividade (GUÉNÉGO et al., 2007), porém, no presente relato, o paciente era de pequeno porte e, portanto, menos susceptível a complicações dessa técnica. Para CASALE \& MCCARTHY (2009), são necessárias informações adicionais sobre os tipos e frequência de complicações associadas à técnica de SFT. Nesse relato, o orifício distal criado para estabilização de RLCCr encontra-se na região diafisária da tíbia, mais estreita que a região da crista tibial, portanto, é possível supor que a fratura tenha ocorrido devido à maior fragilidade da região, o que poderia ter sido evitado se o orifício fosse perfurado dorsal à região mais proeminente da crista da tíbia, como recomendado em literatura recente (JOHNSON \& DUNNING, 2005).

Com a ocorrência da fratura de tíbia, o fio da SFT foi removido e esta foi tratada com placa e parafusos, sendo esta uma das técnicas recomendadas para osteossíntese de tíbia (SEAMAN \& SIMPSON, 2004). A escolha do método de fixação baseia-se no tipo e localização da fratura, tamanho e idade do animal e número de ossos envolvidos (DE YOUNG \& PROBST, 1993). Diversos métodos de fixação foram propostos para o tratamento de fraturas na tíbia (DE YOUNG \& PROBST, 1993), como fixação esquelética externa linear ou circular e placa (SEAMAN \& SIMPSON, 2004). A osteossíntese com FEEC, utilizada neste caso, destacase por apresentar grande resistência às forças de torção e arqueamento, porém é axialmente elástico, o que difere de sistemas rígidos (LEWIS et al., 1998).

A fratura foi corrigida com FEEC e, embora BILGILI (2004) tenha afirmado que o ideal é a colocação de dois anéis proximais e dois distais à fratura, optou- se por colocar um anel proximal, devido ao tamanho pequeno do fragmento e do cão. $O$ paciente apresentou apoio parcial do membro quatro dias após o procedimento cirúrgico e deambulação normal com 90 dias de PO, sendo o retorno funcional precoce um dos objetivos da redução de fraturas (DE YOUNG \& PROBST, 1993).

A fixação esquelética externa circular pode ser eficaz e permitir apoio precoce e consolidação da fratura de tíbia decorrente de complicação da técnica de sutura fabelo-tibial.

\section{REFERÊNCIAS}

BILGILI, H. Circular external fixation system of Ilizarov: part V. Fracture treatment by the Ilizarov technique. Veteriner Cerrahi Dergisi, v.10, n.1-2, p.75-89, 2004. Disponível em: 〈http://www.vetcer.org/75_89_9412.pdf>. Acesso em: 6 out. 2012.

BUQUERA, L.E.C. et al. Estabilização extra-articular do joelho com fáscia lata autógena em cães de raças de grande porte. Arquivo Brasileiro de Medicina Veterinária e Zootecnia, v.59, n.3, p.685-694, 2007. Disponível em: <http://www.scielo.br/pdf/ abmvz/v59n3/a21v59n3.pdf>. Acesso em: 20 ago. 2011. doi: 10.1590/S0102-09352007000300021.

CASALE, S.A.; MCCARTHY, R.J. Complications associated with lateral fabellotibial suture surgery for cranial cruciate ligament injury in dogs: 363 cases (1997-2005). Journal of the American Veterinary Medical Association, v.234, n.2, p.229-235, 2009. Disponível em: <http://avmajournals.avma.org/doi/pdf/10.2460/ javma.234.2.229>. Acesso em: 6 out. 2012. doi: 10.2460/ javma.234.2.229.

CHAUVET, A.E. et al. Evaluation of fibular head transposition, lateral fabellar suture, and conservative treatment of cranial cruciate ligament rupture in large dogs: a retrospective study. Journal of American Animal Hospital Association, v.32, n. 3, p. 247-255, 1996.

DE YOUNG, D.J., PROBST, C. Methods of internal fracture fixation. In: SLATTER, D. Textbook of small animal surgery. Philadelphia: Saunders, 1993. V.2, cap.122, p.1610-1631.

DULISCH, M.L. Suture reaction following extra-articular stifle stabilization in the dog. Part 1: a prospective study of 66 stifles. Journal of American Animal Hospital Association, v.17, n. 4 , p. $569-571,1981$.

GAMBARDELLA, P.C. et al. Lateral suture technique for management of anterior cruciate ligament rupture in dogs a retrospective study. Journal of American Veterinary Medical Association, v.178, n.1, p.33-38, 1981.

GUÉNÉGO, L. et al. Cranial cruciate ligament rupture in large and giant dogs - A retrospective evaluation of a modified lateral extracapsular stabilization. Veterinary and Comparative Orthopaedics and Traumatology, v.20,n.1, p.43-50, 2007. Disponível em: <http://www.schattauer.de/en/magazine/subjectareas/journals-z/vcot/contents/archive/issue/ 134/manuscript/ 7842.html>. Acesso em: 20 ago. 2011. 
HALLING, K.B.et al. Use of circular external skeletal fixator constructs to stabilize tarsometatarsal arthrodeses in three dogs. Veterinary and Comparative Orthopaedics and Traumatology, v.17, n.4, p.204-209, 2004.

JOHNSON, A.L. et al. Cranial cruciate repair with a lateral fabellar suture. In: JOHNSON, A.L. et al. Atlas of orthopedic surgical procedures of the dog and cat. Missouri: Elsevier, 2005. Cap.21, p.60-63.

JOHNSON, J.A. et al. Incidence of canine appendicular musculosketal disorders in 16 veterinary teaching hospitals from 1980 through 1989. Veterinary and Comparative Orthopaedics and Traumatology, v.7, n.1, p.59-69, 1994.

KIM, S.E. et al. Tibial osteotomies for cranial cruciate ligament in dogs. Veterinary Surgery, v.37, n.2, p.111- 125, 2008. Disponível em: <http://onlinelibrary.wiley.com/doi/10.1111/ j.1532-950X.2007.00361.x/pdf>. Acesso em: 20 ago. 2011. doi: 10.1111/j.1532- 950X.2007.00361.x.

KORVICK, D.L. et al. Surgeons preferences in treating cruciate ligament ruptures in dogs. Journal of the American Veterinary Medical Association, v.205, n.9, p.1318-1324, 1994

LEWIS, D.D. et al. Biomechanics of circular external skeletal fixation. Veterinary Surgery, v.25, n.5, p.454-464, 1998.
Disponível em: <http://onlinelibrary.wiley.com/doi/10.1111/ j.153-950X.1998.tb00156.x/pdf>. Acesso em: 20 ago. 2011. doi: 10.1111/j.1532-950X.1998.tb00156.x.

OLCAY, B.; BILGILI, H. Experimental studies for treatments of tibia fractures in dogs by circular external fixator (Ilizarov apparatus). Journal of the Faculty of Veterinary Medicine University of Yuzuncu Yil, v.10, n.1, p.15-19, 1999.

OWEN, M.A. Use of the Ilizarov method to manage a septic tibial fracture nonunion with a large cortical defect. Journal of Small Animal Practice, v.41, n.3, p.124-127, 2000

SEAMAN, J.A.; SIMPSON, A.M. Tibial fractures. Clinical Techniques in Small Animal Practice, v.19, n.3, p.151167, 2004.

SMITH, B. Viewpoints in surgery cruciate ligament rupture, extracapsular stabilization. Australian Veterinary Journal, v.78, n.6, p.382-383, 2000.

WARZEE, C.C. et al. Effect of the tibial plateau leveling on cranial and caudal tibial thrust in canine cranial cruciate deficient stifles: an in vitro experimental study. Veterinary Surgery, v.30, n.3, p.278-286, 2001. Disponível em: <http:// onlinelibrary.wiley.com/doi/10.1053/jvet.2001.21400/pdf $>$. Acesso em: 20 ago. 2011. doi: 10.1053/jvet.2001.21400. 